\title{
A randomized phase III study of short- course radiotherapy combined with Temozolomide in elderly patients with newly diagnosed glioblastoma; Japan clinical oncology group study JCOG1910 (AgedGlio-PIII)
}

Yoshiki Arakawa ${ }^{1 *}\left(\mathbb{D}\right.$, Keita Sasaki ${ }^{2}$, Yohei Mineharu$^{1}$, Megumi Uto $^{3},{\text { Takashi Mizowaki }{ }^{*},}^{*}$ Junki Mizusawa ${ }^{2}$, Yuta Sekino ${ }^{2}$, Tomohiro Ono ${ }^{3}$, Hidefumi Aoyama ${ }^{4}$, Kaishi Satomi ${ }^{5}$, Koichi Ichimura ${ }^{6}$, Manabu Kinoshita ${ }^{7}$, Makoto Ohno ${ }^{8}$, Yoshinori Ito ${ }^{9}$, Ryo Nishikawa ${ }^{10}$, Haruhiko Fukuda ${ }^{2}$, Yasumasa Nishimura ${ }^{11}$, Yoshitaka Narita ${ }^{8}$ and Brain Tumor Study Group and Radiation Therapy Study Group of the Japan Clinical Oncology Group

\section{Abstract}

Background: The current standard treatment for elderly patients with newly diagnosed glioblastoma is surgery followed by short-course radiotherapy with temozolomide. In recent studies, 40 Gy in 15 fractions vs. 60 Gy in 30 fractions, $34 \mathrm{~Gy}$ in 10 fractions vs. $60 \mathrm{~Gy}$ in 30 fractions, and $40 \mathrm{~Gy}$ in 15 fractions vs. $25 \mathrm{~Gy}$ in 5 fractions have been reported as non-inferior. The addition of temozolomide increased the survival benefit of radiotherapy with $40 \mathrm{~Gy}$ in 15 fractions. However, the optimal regimen for radiotherapy plus concomitant temozolomide remains unresolved.

\footnotetext{
*Correspondence: yarakawa@kuhp.kyoto-u.ac.jp; mizo@kuhp.kyoto-u.ac.jp 'Department of Neurosurgery, Kyoto University Graduate School of Medicine, 54 Kawaharacho, Shogoin, Sakyo-ku, Kyoto 606-8507, Japan

${ }^{3}$ Department of Radiation Oncology and Image-Applied Therapy, Kyoto

University Graduate School of Medicine, 54 Kawaharacho, Shogoin, Sakyo-ku, Kyoto 606-8507, Japan

Full list of author information is available at the end of the article
}

C C The Author(s). 2021 Open Access This article is licensed under a Creative Commons Attribution 4.0 International License, which permits use, sharing, adaptation, distribution and reproduction in any medium or format, as long as you give appropriate credit to the original author(s) and the source, provide a link to the Creative Commons licence, and indicate if changes were made. The images or other third party material in this article are included in the article's Creative Commons licence, unless indicated otherwise in a credit line to the material. If material is not included in the article's Creative Commons licence and your intended use is not permitted by statutory regulation or exceeds the permitted use, you will need to obtain permission directly from the copyright holder. To view a copy of this licence, visit http://creativecommons.org/licenses/by/4.0/ The Creative Commons Public Domain Dedication waiver (http://creativecommons.org/publicdomain/zero/1.0/) applies to the data made available in this article, unless otherwise stated in a credit line to the data. 


\begin{abstract}
Methods: This multi-institutional randomized phase III trial was commenced to confirm the non-inferiority of radiotherapy comprising 25 Gy in 5 fractions with concomitant $\left(150 \mathrm{mg} / \mathrm{m}^{2} /\right.$ day, 5 days $)$ and adjuvant temozolomide over $40 \mathrm{~Gy}$ in 15 fractions with concomitant $\left(75 \mathrm{mg} / \mathrm{m}^{2} /\right.$ day, every day from first to last day of radiation) and adjuvant temozolomide in terms of overall survival (OS) in elderly patients with newly diagnosed glioblastoma. A total of 270 patients will be accrued from 51 Japanese institutions in 4 years and follow-up will last 2 years. Patients 71 years of age or older, or $71-75$ years old with resection of less than $90 \%$ of the contrastenhanced region, will be registered and randomly assigned to each group with 1:1 allocation. The primary endpoint is OS, and the secondary endpoints are progression-free survival, frequency of adverse events, proportion of Karnofsky performance status preservation, and proportion of health-related quality of life preservation. The Japan Clinical Oncology Group Protocol Review Committee approved this study protocol in April 2020. Ethics approval was granted by the National Cancer Center Hospital Certified Review Board. Patient enrollment began in August 2020.
\end{abstract}

Discussion: If the primary endpoint is met, short-course radiotherapy comprising 25 Gy in 5 fractions with concomitant and adjuvant temozolomide will be a standard of care for elderly patients with newly diagnosed glioblastoma.

Trial registration: Registry number: jRCTs031200099.

Date of Registration: 27/Aug/2020. Date of First Participant Enrollment: 4/Sep/2020.

Keywords: Randomized controlled trial, Glioblastoma, Elderly, Temozolomide, Short-course radiotherapy

\section{Background}

Glioblastoma is an incurable primary brain tumor with a median overall survival (OS) less than 2 years and a 5year survival rate of $7.2-16 \%$, and comprising $12-14.5 \%$ of primary brain tumors $[1,2]$. The incidence of glioblastoma peaks among patients in their 60 s to 70 s and is increasing with the growth of the elderly population [13]. Age is associated with poor prognosis, and median OS is less than 12 months among patients over 70 years old [1]. Initial Karnofsky performance status (KPS) before treatment is lower than 70 in $36 \%$ of patients [1]. Furthermore, the progression of glioblastoma can easily decrease performance status by reducing functional and cognitive ability, particularly among elderly patients.

The present standard treatment for patients younger than 70 years old with glioblastoma is radiotherapy comprising 60 Gy in 30 fractions with concomitant and adjuvant temozolomide [4, 5]. Most clinical trials for glioblastoma have excluded elderly patients because of their poor prognosis, comorbidity and sensitivity of the aging brain to radiation [6]. Several studies of elderly patients with glioblastoma evaluating radiotherapy as 60 Gy in 30 fractions with concomitant and adjuvant temozolomide showed prolonged OS in patients with good performance status but treatment-related toxicities such as greater deterioration of mental status [6-9]. Although conventionally fractionated radiotherapy effectively prolonged survival in elderly patients with glioblastoma, low completion rates due to the long duration of treatment and declines in activities of daily living (ADL) remain concerning. Hypofractionated radiotherapy has thus been developed to preserve efficacy and decrease toxicities during treatment.

In 2017, the Canadian Cancer Clinical Trials Group (CCTG) and the European Organization for Research and Treatment of Cancer (EORTC) reported that median OS was longer with short-course radiotherapy comprising $40 \mathrm{~Gy}$ in 15 fractions plus concomitant $(75 \mathrm{mg}$ / $\mathrm{m}^{2}$, daily from first to last day of radiation) and adjuvant (150-200 $\mathrm{mg} / \mathrm{m}^{2}$, days $1-5$, every 4 weeks, 12 cycles) temozolomide than with 40 Gy in 15 fractions alone (9.3 months vs. 7.6 months), as was median progression-free survival (PFS) (5.3 months vs. 3.9 months) in elderly patients with newly diagnosed glioblastoma (CE.6) [10]. The addition of temozolomide to short-course radiotherapy did not disturb quality of life (QOL) within manageable chemotherapy-related adverse events. However, in a subgroup analysis, patients $65-70$ years old benefited less from the addition of temozolomide than those 71-75 years old or 76 years old or older. In a background with fewer enrolled patients 65 to 70 years old than 71 to 75 years old, patients 65 to 70 years old with satisfactory performance status are generally treated with radiotherapy as $60 \mathrm{~Gy}$ in 30 fractions plus concomitant and adjuvant temozolomide. Based on these considerations, a regimen of radiotherapy as 40 Gy in 15 fractions plus temozolomide could be an appropriate standard treatment for newly diagnosed glioblastoma patients 71 years old or above.

Three other phase III trials were conducted for further hypofractionated radiotherapy in elderly patients with newly diagnosed glioblastoma. Those studies compared 
40 Gy in 15 fractions vs. 60 Gy in 30 fractions [11], 34 Gy in 10 fractions vs. 60 Gy in 30 fractions [12], and 40 Gy in 15 fractions vs. 25 Gy in 5 fractions [13] and reported non-inferior results in terms of safety and efficacy for elderly patients with newly diagnosed glioblastoma (Table 1 [10-15]). However, those studies all had small sample sizes and the results have not been confirmed by other investigations, so the optimal dose and number of fractions remain unclear, particularly when administered in combination with temozolomide.

In August 2020, the Brain Tumor Study Group and the Radiation Therapy Study Group of the Japan Clinical Oncology Group (JCOG) started a multicenter randomized controlled phase III trial in elderly patients with newly diagnosed glioblastoma (JCOG1910, AgedGlioPIII).

\section{Methods / design}

\section{Aim}

The purpose of this study was to confirm the noninferiority of radiotherapy as 25 Gy in 5 fractions with concomitant and adjuvant temozolomide over $40 \mathrm{~Gy}$ in 15 fractions with concomitant and adjuvant temozolomide in terms of OS in elderly patients with newly diagnosed glioblastoma.

\section{Randomization}

The first registration is performed by JCOG Web System to the JCOG Data Center if all eligibility criteria for the first registration are satisfied. Methylation status of the O6-methylguanine-DNA methyltransferase (MGMT) promoter region is examined using surgical specimens before the second registration. After confirming the inclusion criteria for the second registration have been met, the second registration will be made by the JCOG
Web System to the JCOG Data Center. Patients will be randomized in a 1:1 ratio into either arm A (standard treatment arm: radiotherapy as $40 \mathrm{~Gy}$ in 15 fractions with concomitant and adjuvant temozolomide) or arm B (experimental treatment arm: radiotherapy as 25 Gy in 5 fractions with concomitant and adjuvant temozolomide) using a minimization method with a random component while balancing arms with respect to institution, extent of surgical resection (partial resection + total resection vs. biopsy), Karnofsky performance status $(<70 \%$ vs. $\geq 70 \%$ ) and methylation status of the MGMT promoter region (methylated vs. unmethylated).

\section{Study setting}

A multi-institutional, randomized controlled trial.

\section{Funding}

This study is supported by the National Cancer Center Research and Development Fund (2020-J-3) and the Japan Agency for Medical Research and Development (JP20ck0106619).

\section{Endpoints}

The primary endpoint is OS, and secondary endpoints are PFS, frequency of adverse events (AEs), proportion of Karnofsky performance status preservation, and proportion of health-related QOL preservation.

OS is defined as the time from registration to death from any cause, censored as of the last day when the patient is known to be alive. PFS is defined as the time from registration to either the first event of tumor progression or death from any cause, censored as of the latest day when the patient is alive without any evidence of progression. Tumor response is evaluated according to the Response Assessment in Neuro-Oncology (RANO)

Table 1 Randomized controlled trials in elderly patients with glioblastoma

\begin{tabular}{|c|c|c|c|c|c|}
\hline Authors, publication year, study name & Age cut-off, years & Number & Intervention & mPFS, months & mOS, months \\
\hline \multirow[t]{2}{*}{ Roa et al., 2004 [11] } & $\geq 60$ & 47 & $60 \mathrm{~Gy} / 30 \mathrm{fr}$ & N/A & 5.1 \\
\hline & & 48 & $40 \mathrm{~Gy} / 15 \mathrm{fr}$ & N/A & 5.6 \\
\hline \multirow[t]{2}{*}{ Keime-Guibert et al., 2007 [14], ANOCEF } & $\geq 70$ & 42 & Best supportive care & 1.2 & 3.9 \\
\hline & & 39 & $50 \mathrm{~Gy} / 28 \mathrm{fr}$ & 3.4 & 6.7 \\
\hline \multirow[t]{2}{*}{ Wick et al., 2012 [15], NOA-08 } & $>65$ & 178 & $60 \mathrm{~Gy} / 30 \mathrm{fr}$ & 4.7 & 9.6 \\
\hline & & 195 & Dose-dense temozolomide & 3.3 & 8.6 \\
\hline \multirow[t]{3}{*}{ Malmström et al., 2012 [12], Nordic study } & $>70$ & 100 & $60 \mathrm{~Gy} / 30 \mathrm{fr}$ & N/A & 6 \\
\hline & & 98 & $34 \mathrm{~Gy} / 10 \mathrm{fr}$ & N/A & 7.5 \\
\hline & & 93 & Temozolomide & N/A & 8.3 \\
\hline \multirow[t]{2}{*}{ Roa et al., 2015 [13] } & $\geq 65$ & 50 & $40 \mathrm{~Gy} / 15 \mathrm{fr}$ & 4.2 & 6.4 \\
\hline & & 48 & $25 \mathrm{~Gy} / 5 \mathrm{fr}$ & 4.2 & 7.9 \\
\hline \multirow[t]{2}{*}{ Perry et al., 2017 [10], CE.6 } & $\geq 65$ & 281 & $40 \mathrm{~Gy} / 15 \mathrm{fr}$ & 3.9 & 7.6 \\
\hline & & 281 & $40 \mathrm{~Gy} / 15 \mathrm{fr}+$ temozolomide & 5.3 & 9.3 \\
\hline
\end{tabular}


criteria [16]. AEs are evaluated according to the Common Terminology Criteria for Adverse Events version 5.0 (CTCAE 5.0). SAE is defined as any $\geq$ grade 4 non-hematological toxicity at least possibly related to treatment, unexpected $\mathrm{AE}$ requiring hospitalization or prolongation of hospital stay, death from any cause during treatment or within 30 days after a protocol treatment, or treatment-related death. Health-related QOL is measured in accordance with EORTC QLQ-C30 and EORTC QLQ-BN20.

\section{Inclusion criteria}

First registration criteria:

(1) Tumor diagnosed as glioblastoma or Grade III glioma (WHO 2016 criteria) in pathological diagnosis during surgery, or histologically proven glioblastoma diagnosed according to WHO 2016 criteria.

(2) Tumor specimen available for analysis of MGMT promoter methylation status.

(3) No history of treatments for glioma except for resection including biopsy; in that case, second resection within 21 days after first resection.

(4) First registration within 21 days after surgery.

(5) Tumor present in the supratentorial region on preoperative contrast-enhanced MRI of the brain.

(6) Preoperative contrast-enhanced MRI of the brain reveals no dissemination.

(7) Presence of a measurable lesion is not mandatory.

(8) Patient 71 years old or older at registration; 71 to 75 years old with resection of less than $90 \%$ of contrast-enhanced region.

(9) ECOG performance status (PS) of 0, 1, 2, or 3 due to neurological symptoms caused by the tumor.

(10)No prior chemotherapy or radiation therapy to the brain for any intracranial tumors.

(11)Sufficient organ function.

(i) Neutrophil count $\geq 1500 / \mathrm{mm}^{3}$.

(ii) Hemoglobin $\geq 8.0 \mathrm{mg} / \mathrm{dl}$.

(iii) Platelet count $\geq 100,000 / \mathrm{mm}^{3}$.

(iv) $\mathrm{AST} \leq 120 \mathrm{U} / \mathrm{L}$.

(v) $\mathrm{ALT} \leq 120 \mathrm{U} / \mathrm{L}$.

(vi) $\mathrm{Cr} \leq 1.605 \mathrm{mg} / \mathrm{dL}$ for males, or $\leq 1.185 \mathrm{mg} / \mathrm{dL}$ for females.

(12)Informed consent provided voluntarily by the patient.
Second registration criteria:

(1) Second registration within 21 days of the first registration.

(2) Histologically proven glioblastoma diagnosed according to WHO 2016 criteria.

(3) Confirmation of methylation or unmethylation in MGMT promoter region.

Exclusion criteria

(1) Synchronous or metachronous malignancies (within the preceding 2 years).

(2) Infections needing systemic treatment at time of registration.

(3) Body temperature above $38^{\circ} \mathrm{C}$ at registration.

(4) Severe psychological disease at time of registration.

(5) Continuous systemic corticosteroid or immunosuppressant treatment due to diseases other than brain tumor.

(6) Uncontrollable diabetes mellitus.

(7) Unstable angina pectoris, or history of myocardial infarction within the preceding 6 months.

(8) Interstitial pneumonia, pulmonary fibrosis, or severe emphysema at time of registration.

(9) Gadolinium allergy.

(10)Positive results for HIV antibody.

(11)Positive results for HBs antigen.

\section{Interventions}

\section{Arm A:}

(1) 40 Gy in 15 daily fractions with temozolomide.

(i) Concomitant phase, temozolomide $\left(75 \mathrm{mg} / \mathrm{m}^{2}\right.$, daily from first to last day of radiation), radiation $(2.67 \mathrm{~Gy} /$ day, 5 days/week, 15 times and 40 Gy in total)

(ii) Maintenance phase, temozolomide (150-200 mg/ $\mathrm{m}^{2}$, days $1-5$, every 4 weeks) 12 cycles.

\section{Arm B:}

(1) 25 Gy in 5 daily fractions with temozolomide.

(i) Concomitant phase, temozolomide $\left(150 \mathrm{mg} / \mathrm{m}^{2}, 5\right.$ days from first day), radiation ( $5 \mathrm{~Gy} /$ day, 5 days/week, 5 times and 25 Gy in total)

(ii) Maintenance phase, temozolomide (150-200 mg/ $\mathrm{m}^{2}$, days $1-5$, every 4 weeks) 12 cycles.

\section{Procedures of radiotherapy}

Contouring was performed using computed tomography (CT) with a maximum slice thickness of $5 \mathrm{~mm}$. Gross tumor volume (GTV) was defined as residual tumor according to pre- and postoperative contrast-enhanced MRI. Clinical target volume (CTV) was created by adding $1.5-\mathrm{cm}$ margins to the GTV and resection cavity. In addition, CTV included surrounding edema (high- 
intensity area on T2-weighted or fluid-attenuated inversion recovery images). The margin for the planning target volume was set at $3-5 \mathrm{~mm}$. The same target setting was used for Arms A and B.

\section{Follow-up}

All patients will be followed-up for at least 2 years after completion of protocol treatment. Gadolinium-enhanced MRI of the brain will be evaluated at least every 8 weeks until disease progression or death. Tumor response will also be assessed every 8 weeks according to RANO criteria. Physical examination, laboratory tests, and evaluation of AEs according to CTCAE version 5.0 will be carried out at least every 4 weeks during protocol treatment.

\section{Statistical analysis}

This study is designed as a multi-institutional, randomized controlled trial to confirm the non-inferiority of radiotherapy of $25 \mathrm{~Gy}$ in 5 fractions with concomitant and adjuvant temozolomide over $40 \mathrm{~Gy}$ in 15 fractions with concomitant and adjuvant temozolomide in terms of OS in elderly patients with newly diagnosed glioblastoma. The required sample size for randomization is calculated as 264 , which will provide $70 \%$ power with a hypothesized 1-year survival rate of $37.8 \%$ in both arms and a non-inferiority margin of 1.32 using a one-sided alpha of 0.05 to observe 249 deaths. Considering some patients will not be registered due to progression before the second registration, the planned sample size for the first registration is 270 patients in a 4-year accrual period and 2-year follow-up period. Stratified Cox regression analysis with extent of surgical resection, Karnofsky performance status and methylation status of the MGMT promoter region will be performed for primary analysis.

\section{Interim analysis and monitoring}

We plan to conduct an interim analysis to judge whether the study should be terminated early due to futility or clear evidence of efficacy. The interim analysis will be conducted after half of the planned number patients have been enrolled. The Lan-DeMets method with an O'Brien and Fleming-type alpha spending function will be used to adjust the multiplicity of the interim analysis and the primary analysis [17].

JCOG Data Center staff will conduct central monitoring issuing a monitoring report twice annually to evaluate and improve study progress, data integrity and patient safety. Futility will be considered at each monitoring report if necessary. For quality assurance, sitevisit audits, not for a study-specific basis but for the study group basis, will be performed by the JCOG Audit Committee.

\section{Discussion}

Reducing the treatment burden while maintaining efficacy is important for elderly patients with newly diagnosed glioblastoma, which shows poor prognosis. The disadvantages of the standard treatment are prolonged gastrointestinal symptoms caused by temozolomide, prolonged hospitalization for about 2 months, and deterioration of QOL due to hospitalization. This study therefore plans to confirm whether hypofractionated radiotherapy requiring a shorter treatment period with temozolomide can overcome the disadvantages of standard treatment without compromising efficacy.

Based on the CE. 6 trial results, the regimen of radiotherapy comprising 40 Gy in 15 fractions plus temozolomide is the standard treatment for newly diagnosed glioblastoma patients 65 years old or older. In actual clinical practice, however, elderly patients with satisfactory performance status, particularly those under 75 years old, tend to be treated with $60 \mathrm{~Gy}$ of radiotherapy in 30 fractions plus concomitant and adjuvant temozolomide. Our other phase III trial, JCOG1703, began in June 2019 and is currently recruiting patients under 75 years old with newly diagnosed glioblastoma to confirm the superiority of maximal resection with carmustine wafer implantation followed by chemoradiotherapy with temozolomide over maximal resection followed by chemoradiotherapy with temozolomide, at least in terms of OS [18]. As the definition of maximal tumor resection is resection exceeding $90 \%$, patients with $71-75$ years and resection of less than $90 \%$ of the contrast-enhanced region has been set as an inclusion criterion for this study.

$M G M T$ is a DNA repair enzyme that confers chemoresistance to alkylating agents [19]. Promoter methylation of MGMT reflects epigenetic silencing associated with longer survival in patients with glioblastoma treated using alkylating agents. In the analysis of the CE. 3 trial, patients with a methylated $M G M T$ promoter showed a survival benefit from temozolomide (median OS, 21.7 months vs. 15.3 months), whereas those with an unmethylated MGMT promoter did not (median OS, 12.7 months vs. 11.8 months) [20]. Furthermore, MGMT promoter methylation represents an independent favorable prognostic factor irrespective of treatment. The CE.6 trial showed a similar result that patients with a methylated MGMT promoter achieved significantly prolonged survival with temozolomide (median OS, 13.5 months with radiotherapy plus temozolomide vs. 7.7 months with radiotherapy alone) and those with unmethylated MGMT promoter benefited slightly from temozolomide (median OS, 10.0 months with radiotherapy plus temozolomide vs. 7.9 months with radiotherapy alone) [10], which may suggest that short-course radiotherapy increases the efficacy of temozolomide in glioblastomas with unmethylated MGMT promoter. 
Table 2 Equivalent dose in 2-Gy fractions (EQD2) estimated on the LQ model

\begin{tabular}{|c|c|c|c|c|c|}
\hline \multirow[b]{2}{*}{ Clinical trials } & \multirow[b]{2}{*}{ Radiation dose/fractions } & \multicolumn{4}{|c|}{$a / \beta$ value } \\
\hline & & 1.2 & 3.0 & 5.6 & 10.0 \\
\hline Conventional radiotherapy & $60 \mathrm{~Gy} / 30 \mathrm{fr}$ & 60.0 & 60.0 & 60.0 & 60.0 \\
\hline Perry et al., 2017 [10], CE.6 & $40 \mathrm{~Gy} / 15 \mathrm{fr}$ & 48.4 & 45.4 & 43.6 & 42.3 \\
\hline Roa et al., 2015 [13] & $25 \mathrm{~Gy} / 5 \mathrm{fr}$ & 48.4 & 40.0 & 34.9 & 31.3 \\
\hline Malmström et al., 2012 [12], Nordic study & $34 \mathrm{~Gy} / 10 \mathrm{fr}$ & 48.9 & 43.5 & 40.3 & 38.0 \\
\hline
\end{tabular}

Radiotherapeutic regimens of 40 Gy in 15 fractions, 34 Gy in 10 fractions, and 25 Gy in 5 fractions have been shown to offer similar efficacy and safety for elderly patients with glioblastoma according to the three earlier phase III studies [11-13]. The current task is to determine the optimal dose and number of fractions, particularly in combination with temozolomide, for elderly patients with newly diagnosed glioblastoma. The linearquadratic (LQ) model has been used for predicting radiobiological response [21]. Equivalent-dose fractionation can be estimated by calculating equivalent doses in 2-Gy fractions (EQD2) on the LQ model. Although several studies have shown that the $\alpha / \beta$ values of glioma and normal brain tissue were 5-10 Gy and 2-3 Gy, respectively [22-24], and the $\alpha / \beta$ value of glioblastoma in elderly patients is estimated as less than $1.2 \mathrm{~Gy}$ from the results of recent clinical trials $[10,12,13]$ (Table 2). On the basis of the estimated $\alpha / \beta$ value $1.2 \mathrm{~Gy}$, EQD2s of 40 Gy in 15 fractions and 25 Gy in 5 fractions were considered equivalent for glioblastoma in elderly patients, suggesting similar efficacy. Meanwhile, the EQD2 of 25 Gy in 5 fractions is less than that of $40 \mathrm{~Gy}$ in 15 fractions for normal brain tissue, meaning less damage would be incurred by 25 Gy in 5 fractions. These estimates are supported by study results finding no differences in OS, PFS, or QOL between patients receiving the two radiotherapy regimens of $40 \mathrm{~Gy}$ in 15 fractions and $25 \mathrm{~Gy}$ in 5 fractions [13].

As concomitant treatment in the standard treatment, temozolomide is administered at $75 \mathrm{mg} / \mathrm{m}^{2}$ for 21 days, resulting in a total dose of $1575 \mathrm{mg} / \mathrm{m}^{2}$. On the other hand, if temozolomide at $75 \mathrm{mg} / \mathrm{m}^{2}$ is adopted as the concomitant treatment, the total dose of temozolomide is considerably reduced to $375 \mathrm{mg} / \mathrm{m}^{2}$ in 5 days and treatment intensity may thus be insufficient. Administration of temozolomide at $150 \mathrm{mg} / \mathrm{m}^{2}$ for 5 days has been confirmed as safe and effective as the first administration of maintenance treatment in the previous report [25]. In the study treatment, a synergistic effect is expected because both the single dose of temozolomide and radiation are increased, and maintenance treatment is implemented 2 weeks earlier than that in the standard treatment, so that the efficacy of temozolomide treatment is considered to be enhanced. According to the above, temozolomide at $150 \mathrm{mg} / \mathrm{m}^{2} /$ day administered for
5 days was adopted as concomitant treatment for the study treatment.

Based on the prediction of radiobiological response from the results of clinical trials, the aim of this study is to confirm the non-inferiority of radiotherapy comprising 25 Gy in 5 fractions with concomitant and adjuvant temozolomide over 40 Gy in 15 fractions with concomitant and adjuvant temozolomide in terms of OS among elderly patients with newly diagnosed glioblastoma. If the primary endpoint is met, radiotherapy as 25 Gy in 5 fractions with concomitant and adjuvant temozolomide will be established as a standard of care for elderly patients with newly diagnosed glioblastoma. Furthermore, the $\alpha / \beta$ value of glioblastoma in elderly patients will be confirmed as less than $1.2 \mathrm{~Gy}$. This study is designed as a multi-institutional, randomized controlled trial with a non-inferiority margin of 1.32 , considering the disease rarity.

\section{Abbreviations}

ADL: Activities of daily living; AEs: Adverse events; CCTG: Canadian Cancer Clinical Trials Group; Cl: Confidence interval; CTCAE: Common Terminology Criteria for Adverse Events; ECOG: Eastern Cooperative Oncology Group; EORTC: European Organisation for Research and Treatment of Cancer; EQD2: Equivalent dose in 2-Gy fractions; JCOG: Japan Clinical Oncology Group; KPS: Karnofsky performance status; LQ: Linear-quadratic; MGMT: O6methylguanine-DNA methyltransferase; MR: Magnetic resonance; OS: Overall survival; PFS: Progression-free survival; PS: Performance status; QOL: Quality of life; RANO: Response Assessment in Neuro-Oncology; SAE: Serious adverse event; SPIRIT: Standard Protocol Items: Recommendations for Interventional Trials

\section{Acknowledgements}

We would like to thank all members of the JCOG Data Center/Operation office, Brain Tumor Study Group and Radiation Therapy Study Group of the Japan Clinical Oncology Group, and Ms. Keiko Furukawa from the Kyoto University Hospital Cancer Center.

\section{Consortia}

The Brain Tumor Study Group and the Radiation Therapy Study Group of the Japan Clinical Oncology Group.

\section{Authors' contributions}

YA and TM, as the corresponding author, wrote the manuscript. YA, YM, MU, and TM proposed the concept and idea for JCOG1910 study and drafted the protocol design for the study. KS, JM, YS, and HF contributed to the design and logistics of the protocol, proofread the manuscript, and will undertake statistical analyses. HA, KS, and IK contributed to the design of central diagnosis in MRI diagnosis, histological diagnosis, and molecular diagnosis, respectively. TO, MK, MO, YI, RN, YN, and YN contributed to the design and logistics of the protocol and conducted initiation of the study. All authors critically revised the manuscript for intellectual content and approved the final manuscript. 


\section{Funding}

This study is supported by the National Cancer Center Research and Development Fund (2020-J-3) and the Japan Agency for Medical Research and Development (AMED, JP20ck0106619).

\section{Availability of data and materials}

Data sharing is not applicable for this article, as no datasets have been generated or analyzed at the time of submission.

\section{Declarations}

\section{Ethics approval and consent to participate}

This study is being conducted in accordance with the principles expressed in the Declaration of Helsinki, Clinical Trials Act (Act No. 16 of April 14, 2017) and SPIRIT (Standard Protocol Items: Recommendations for Interventional Trials) guidelines. The study protocol was approved by the JCOG Protocol Review Committee on April 24th, 2020 and by the National Cancer Center Hospital Certified Review Board on June 25th, 2020 (CRB3180008). Written informed consent has been obtained from all enrolled participants. At the time of submission (May 2021), a total of 51 institutions are participating in this trial. The participating institutions are as follows: Hokkaido University Hospital, Nakamura Memorial Hospital, Sapporo Medical University, Hirosaki University Hospital, Iwate Medical University, Tohoku University Hospital, Yamagata University Hospital, Faculty of Medicine, University of Tsukuba, Jichi Medical University Hospital, Dokkyo Medical University Hospital, Gunma University Hospital, Saitama Cancer Center, Saitama Medical University International Medical Center, Chiba University, Graduate School of Medicine, National Cancer Center Hospital, Nihon University Itabashi Hospital, Kyorin University Faculty of Medicine, Tokyo Metropolitan Cancer and Infectious Diseases Center Komagome Hospital, Tokyo Women's Medical University, Keio University Hospital, Showa University Hospital, Tokyo Medical and Dental University Hospital, The University of Tokyo Hospital, Tokai University Hospital, Juntendo University Hospital, Nippon Medical School Hospital, Yokohama City University Hospital, Kitasato University School of Medicine, Niigata University Medical and Dental Hospital, University of Yamanashi Faculty of Medicine University Hospital, Hamamatsu University Hospital, Shizuoka Cancer Center, Nagoya University School of Medicine, Fujita Health University, University Hospital Kyoto Prefectural University of Medicine, Kyoto University Hospital, Osaka University Graduate School of Medicine, Osaka International Cancer Institute, Kobe University Graduate School of Medicine, Kobe City Medical Center General Hospital, Nara Medical University Hospital, Okayama University Hospital, Hiroshima University Hospital, Ehime University Hospital, Kurume University Hospital, Kyushu University Hospital, Saga University Hospital, Nagasaki University Hospital, Kumamoto University Medical School, Oita University Faculty of Medicine, and Kagoshima University Hospital.

\section{Consent for publication}

Not applicable.

\section{Competing interests}

All authors declare no competing interests in relation to this study protocol.

\section{Author details}

${ }^{1}$ Department of Neurosurgery, Kyoto University Graduate School of Medicine, 54 Kawaharacho, Shogoin, Sakyo-ku, Kyoto 606-8507, Japan. ${ }^{2}$ JCOG Data Center/Operations Office, National Cancer Center Hospital, Tokyo, Japan. ${ }^{3}$ Department of Radiation Oncology and Image-Applied Therapy, Kyoto University Graduate School of Medicine, 54 Kawaharacho, Shogoin, Sakyo-ku, Kyoto 606-8507, Japan. ${ }^{4}$ Department of Radiation Oncology, Faculty of Medicine, Hokkaido University, Sapporo, Hokkaido, Japan. ${ }^{5}$ Department of Diagnostic Pathology, National Cancer Center Hospital, Tokyo, Japan. ${ }^{6}$ Department of Brain Disease Translational Research, Juntendo University Faculty of Medicine, Tokyo, Japan. ${ }^{7}$ Department of Neurosurgery, Asahikawa Medical University, Asahikawa, Hokkaido, Japan. ${ }^{8}$ Department of Neurosurgery and Neuro-Oncology, National Cancer Center Hospital, Tokyo, Japan. ${ }^{9}$ Department of Radiation Oncology, Showa University Graduate School of Medicine, Tokyo, Japan. ${ }^{10}$ Department of Neuro-Oncology/ Neurosurgery, Saitama Medical University International Medical Center, Saitama, Japan. ${ }^{11}$ Department of Radiation Oncology, Kindai University Faculty of Medicine, Osaka, Japan.
Received: 28 July 2021 Accepted: 4 October 2021

Published online: 15 October 2021

\section{References}

1. Report of Brain Tumor Registry of Japan (2005-2008) 14th Edition. Neurol Med Chir (Tokyo). 2017:57(Suppl 1):9-102.

2. Ostrom QT, Patil N, Cioffi G, Waite K, Kruchko C, Barnholtz-Sloan JS. CBTRUS Statistical Report: Primary Brain and Other Central Nervous System Tumors Diagnosed in the United States in 2013-2017. Neuro-oncology. 2020;22(12 Suppl 2):iv1-iv96.

3. Report of Brain Tumor Registry of Japan (2001-2004) 13th Edition.. Neurol Med Chir (Tokyo). 2014;54(Supplement-1):9-102.

4. Stupp R, Hegi ME, Mason WP, van den Bent MJ, Taphoorn MJ, Janzer RC, et al. Effects of radiotherapy with concomitant and adjuvant temozolomide versus radiotherapy alone on survival in glioblastoma in a randomised phase III study: 5-year analysis of the EORTC-NCIC trial. Lancet Oncol. 2009; 10(5):459-66. https://doi.org/10.1016/S1470-2045(09)70025-7.

5. Stupp R, Mason WP, van den Bent MJ, Weller M, Fisher B, Taphoorn MJ, et al. Radiotherapy plus concomitant and adjuvant temozolomide for glioblastoma. N Engl J Med. 2005;352(10):987-96. https://doi.org/10.1056/ NEJMoa043330.

6. Brandes AA, Franceschi E, Tosoni A, Benevento F, Scopece L, Mazzocchi V, et al. Temozolomide concomitant and adjuvant to radiotherapy in elderly patients with glioblastoma: correlation with MGMT promoter methylation status. Cancer. 2009;115(15):3512-8. https://doi.org/10.1002/cncr.24406.

7. Brandes AA, Vastola F, Basso U, Berti F, Pinna G, Rotilio A, et al. A prospective study on glioblastoma in the elderly. Cancer. 2003;97(3):657-62. https://doi.org/10.1002/cncr.11097.

8. Minniti G, De Sanctis V, Muni R, Filippone F, Bozzao A, Valeriani M, et al. Radiotherapy plus concomitant and adjuvant temozolomide for glioblastoma in elderly patients. J Neuro-Oncol. 2008;88(1):97-103. https:// doi.org/10.1007/s11060-008-9538-0.

9. Fiorica F, Berretta M, Colosimo C, Stefanelli A, Ursino S, Zanet E, et al. Glioblastoma in elderly patients: safety and efficacy of adjuvant radiotherapy with concomitant temozolomide. Arch Gerontol Geriatr. 2010;51(1):31-5. https://doi.org/10.1016/j.archger.2009.06.011.

10. Perry JR, Laperriere N, O'Callaghan CJ, Brandes AA, Menten J, Phillips C, et al. Short-course radiation plus Temozolomide in elderly patients with glioblastoma. New Engl J Med. 2017;376(11):1027-37. https://doi.org/10.1 056/NEJMoa1611977.

11. Roa W, Brasher PM, Bauman G, Anthes M, Bruera E, Chan A, et al. Abbreviated course of radiation therapy in older patients with glioblastoma multiforme: a prospective randomized clinical trial. J Clin Oncol. 2004;22(9): 1583-8. https://doi.org/10.1200/JCO.2004.06.082.

12. Malmstrom A, Gronberg BH, Marosi C, Stupp R, Frappaz D, Schultz H, et al. Temozolomide versus standard 6-week radiotherapy versus hypofractionated radiotherapy in patients older than 60 years with glioblastoma: the Nordic randomised, phase 3 trial. Lancet Oncol. 2012; 13(9):916-26. https://doi.org/10.1016/S1470-2045(12)70265-6.

13. Roa W, Kepka L, Kumar N, Sinaika V, Matiello J, Lomidze D, et al. International Atomic Energy Agency Randomized Phase III Study of Radiation Therapy in Elderly and/or Frail Patients With Newly Diagnosed Glioblastoma Multiforme. J Clin Oncol. 2015;33(35):4145-50.

14. Keime-Guibert F, Chinot O, Taillandier L, Cartalat-Carel S, Frenay M, Kantor G, et al. Radiotherapy for glioblastoma in the elderly. New Engl J Med. 2007; 356(15):1527-35. https://doi.org/10.1056/NEJMoa065901.

15. Wick W, Platten M, Meisner C, Felsberg J, Tabatabai G, Simon M, et al. Temozolomide chemotherapy alone versus radiotherapy alone for malignant astrocytoma in the elderly: the NOA-08 randomised, phase 3 trial. Lancet Oncol. 2012;13(7):707-15. https://doi.org/10.1016/S1470-2045(12)701 $64-X$.

16. Wen PY, Macdonald DR, Reardon DA, Cloughesy TF, Sorensen AG, Galanis E, et al. Updated response assessment criteria for high-grade gliomas: response assessment in neuro-oncology working group. Journal Clin Oncol. 2010;28(11):1963-72. https://doi.org/10.1200/JCO.2009.26.3541.

17. Lan KKG, Demets DL. Discrete sequential boundaries for clinical-trials. Biometrika. 1983;70(3):659-63. https://doi.org/10.2307/2336502.

18. Kadota T, Saito R, Kumabe T, Mizusawa J, Katayama H, Sumi M, et al. A multicenter randomized phase III study for newly diagnosed maximally resected glioblastoma comparing carmustine wafer implantation followed by chemoradiotherapy with temozolomide with chemoradiotherapy alone; 
Japan clinical oncology group study JCOG1703 (MACS study). Jpn J Clin Oncol. 2019;49(12):1172-5. https://doi.org/10.1093/jjco/hyz169.

19. Esteller M, Garcia-Foncillas J, Andion E, Goodman SN, Hidalgo OF, Vanaclocha $\mathrm{V}$, et al. Inactivation of the DNA-repair gene MGMT and the clinical response of gliomas to alkylating agents. N Engl J Med. 2000; 343(19):1350-4. https://doi.org/10.1056/NEJM200011093431901.

20. Hegi ME, Diserens AC, Gorlia T, Hamou MF, de Tribolet N, Weller M, et al. MGMT gene silencing and benefit from temozolomide in glioblastoma. N Engl J Med. 2005;352(10):997-1003. https://doi.org/10.1056/NEJMoa043331.

21. van Leeuwen CM, Oei AL, Crezee J, Bel A, Franken NAP, Stalpers $\perp A$, et al. The alfa and beta of tumours: a review of parameters of the linear-quadratic model, derived from clinical radiotherapy studies. Radiat Oncol. 2018;13(1): 96. https://doi.org/10.1186/s13014-018-1040-z.

22. Barazzuol L, Burnet NG, Jena R, Jones B, Jefferies SJ, Kirkby NF. A mathematical model of brain tumour response to radiotherapy and chemotherapy considering radiobiological aspects. J Theor Biol. 2010;262(3): 553-65. https://doi.org/10.1016/j.jtbi.2009.10.021.

23. Jones B, Sanghera P. Estimation of radiobiologic parameters and equivalent radiation dose of cytotoxic chemotherapy in malignant glioma. Int I Radiat Oncol Biol Phys. 2007;68(2):441-8. https://doi.org/10.1016/j.jirobp.2006.12.02 5.

24. Qi XS, Schultz CJ, Li XA. An estimation of radiobiologic parameters from clinical outcomes for radiation treatment planning of brain tumor. Int J Radiat Oncol Biol Phys. 2006;64(5):1570-80. https://doi.org/10.1016/j.jijobp.2 005.12.022.

25. Gallego Perez-Larraya J, Ducray F, Chinot O, Catry-Thomas I, Taillandier L, Guillamo JS, et al. Temozolomide in elderly patients with newly diagnosed glioblastoma and poor performance status: an ANOCEF phase II trial. J Clin Oncol. 2011;29(22):3050-5. https://doi.org/10.1200/JCO.2011.34.8086.

\section{Publisher's Note}

Springer Nature remains neutral with regard to jurisdictional claims in published maps and institutional affiliations.

Ready to submit your research? Choose BMC and benefit from:

- fast, convenient online submission

- thorough peer review by experienced researchers in your field

- rapid publication on acceptance

- support for research data, including large and complex data types

- gold Open Access which fosters wider collaboration and increased citations

- maximum visibility for your research: over $100 \mathrm{M}$ website views per year

At $\mathrm{BMC}$, research is always in progress.

Learn more biomedcentral.com/submissions 